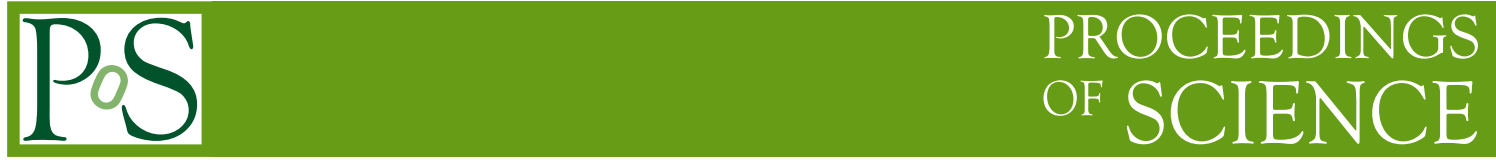

Joey Huston ${ }^{1} 2$

E-mail: huston@msu.edu

\title{
PDFs for the LHC
}

This is a brief review of the current recommendations of the PDF4LHC working group for the use of parton distribution functions (PDFs) and of PDF uncertainties at the LHC, and of the current understanding of available PDF sets on which this recommendation is based.

XVIII International Workshop on Deep-Inelastic Scattering and Related Subjects, DIS 2010

April 19-23, 2010

Firenze, Italy

\section{*Speaker.}

${ }^{\dagger}$ This work was done in collaboration with the PDF4LHC working group and is a brief summary of the interim note. 


\section{Introduction}

The LHC experiments are currently producing cross sections from the $7 \mathrm{TeV}$ data, and thus need accurate predictions for these cross sections and their uncertainties at NLO and NNLO. Crucial to the predictions and their uncertainties are the parton distribution functions (PDFs) obtained from global fits to data from deep-inelastic scattering, Drell-Yan and jet data. A number of groups have produced publicly available PDFs using different data sets and analysis frameworks. It is one of the charges of the PDF4LHC working group ${ }^{1}$ to evaluate and understand differences among the PDF sets to be used at the LHC, and to provide a protocol for both experimentalists and theorists to use the PDF sets to calculate central cross sections at the LHC, as well as to evaluate their PDF uncertainty. A note is being prepared, intended to be an interim summary of our level of understanding as the first LHC cross sections at $7 \mathrm{TeV}$ are being produced. This talk was a brief summary of the studies in that note. The note will appear on the PDF4LHC website.

For the purpose of increasing our quantitative understanding of the similarities and differences between available PDF determinations, a benchmarking exercise between the different groups was performed. This exercise was very instructive in understanding many differences in the PDF analyses: different input data, different methodologies and criteria for determining uncertainties, different ways of parametrising PDFs, differenz number of parametrized PDFs, different treatments of heavy quarks, different perturbative orders, different ways of treating $\alpha_{s}$ (as an input or as a fit parameter), different values of physical parameters such as $\alpha_{s}$ itself and heavy quark masses, and more. This exercise was also very instructive in understanding where the PDFs agree and where they disagree: it established a broad agreement of PDFs (and uncertainties) obtained from data sets of comparable size and it singled out relevant instances of disagreement and of dependence of the results on assumptions or methodology.

\section{The PDF4LHC benchmarks}

A benchmarking exercise was carried out to which all PDF groups were invited to participate. This exercise considered only the most up to date published versions of NLO PDFs from 6 groups: ABKM09 [1], [2], CTEQ6.6 [3], GJR08 [4], HERAPDF1.0 [5], MSTW08 [6], NNPDF2.0 [7]. The benchmark cross sections were evaluated at NLO at both 7 and $14 \mathrm{TeV}$. The interim note contains only the $7 \mathrm{TeV}$ predictions.

All of the benchmark processes were to be calculated with the following settings:

1. at NLO in the $\overline{M S}$ scheme; in 5-flavor quark schemes, with the definition of the scheme to be specified by each group

2. at 7 and $14 \mathrm{TeV}$; for the central value predictions, and for $\pm 68 \%$ and $\pm 90 \%$ c.l. PDF uncertainties

3. with and without the $\alpha_{s}$ uncertainties, with the prescription for combining the PDF and $\alpha_{s}$ errors to be specified; repeating the calculation with a central value of $\alpha_{s}\left(m_{Z}\right)$ of 0.119

\footnotetext{
${ }^{1}$ wiki.terascale.de/index.php?title=PDF4LHC_WIKI
} 
To provide some standardization, a gzipped version of MCFM5.7 [8] was prepared by John Campbell, using the specified parameters and exact input files for each process. It was allowable for other codes to be used, but they had to be checked against the MCFM output values.

The processes included in the benchmarking exercise are given below.

1. $W^{+}, W^{-}$and $Z$ cross sections and rapidity distributions including the cross section ratios $W^{+} / W^{-}$and $\left(W^{+}+W^{-}\right) / Z$ and the $W$ asymmetry as a function of rapidity $\left(\left[W^{+}(y)-\right.\right.$ $\left.\left.W^{-}(y)\right] /\left[W^{+}(y)+W^{-}(y)\right]\right)$. The following specifications were made for the $W$ and $Z$ cross sections:

(a) $m_{Z}=91.188 \mathrm{GeV} ; m_{W}=80.398 \mathrm{GeV}$

(b) zero width approximation used

(c) $G_{F}=0.116637 \times 10^{-5} \mathrm{GeV}^{-2} ; \sin ^{2} \theta_{W}=0.2227$; other EW couplings derived using tree level relations

(d) $\mathrm{BR}(Z \rightarrow l l)=0.03366 ; \mathrm{BR}(W \rightarrow l v)=0.1080$

(e) CKM mixing parameters from Eq. 11.27 of the PDG2009 CKM review

(f) scales: $\mu_{R}=\mu_{F}=m_{Z}$ or $m_{W}$

2. $g g \rightarrow$ Higgs total cross sections at NLO The following specifications were made for the Higgs cross section.

(a) $m_{H}=120,180$ and $240 \mathrm{GeV}$

(b) zero Higgs width approximation, no branching ratios taken into account

(c) top loop only, with $m_{\text {top }}=171.3 \mathrm{GeV}$ in $\sigma_{o}$; scales: $\mu_{R}=\mu_{F}=m_{\text {Higgs }}$

3. $t \bar{t}$ cross section at NLO

(a) $m_{\text {top }}=171.3 \mathrm{GeV}$

(b) zero top width approximation, no branching ratios; scales: $\mu_{R}=\mu_{F}=m_{\text {top }}$

The cross sections chosen are all important cross sections at the LHC, for standard model benchmarking for the case of the $W, Z$ and top cross sections, and discovery potential for the case of the Higgs cross sections. Both $q \bar{q}$ and $g g$ initial states are involved. The NLO $W$ and $Z$ cross sections have a small dependence on the value of $\alpha_{s}\left(m_{Z}\right)$, while the dependence is sizeable for both $t \bar{t}$ and Higgs production. The $q \bar{q}$ and $g g$ PDF luminosities, plotted as a function of $\sqrt{\hat{s} / s}$, have different behavior for the different PDF groups. For example, the CTEQ6.6 and HERAPDF1.0 luminosities tend to be higher at low masses for both initial states than MSTW2008 and NNPDF2.0. The relative behaviors also differ at high mass as well. For masses around the $W / Z$ boson mass, the $q \bar{q}$ luminosities are relatively close to each other, leading to similar predictions for $W$ and $Z$ cross sections at $7 \mathrm{TeV}$. The one - sigma error bands for the CTEQ6.6, MSTW2008 and NNPDF2.0 predictions overlap. There is a larger spread of predictions for $t \bar{t}$ and Higgs boson production (for masses of 120,180 and $240 \mathrm{GeV}$ ), both due to the variations of the $g g$ luminosities and to the different values of $\alpha_{s}\left(m_{Z}\right)$ assumed in the different global analyses. In this case, better agreement is obtained if the predictions are shifted to a common value of $\alpha_{s}$. More details are available in the slides and will be available in the note. 


\section{The PDF4LHC recommendation}

\subsection{NLO prescription}

At NLO, the recommendation is to use (at least) predictions from the CTEQ6.6, MSTW2008 and NNPDF2.0 PDFs. There is a desire to keep the number of PDFs recommended for the prescription to a reasonable size, and the predictions from these three PDFs are in reasonable (but not exact) agreement with each other for most of the relevant LHC observables. There are a few caveats. Neither the CTEQ6.6 nor the MSTW2008 PDF sets use the new combined very accurate HERA data sets, which are instead used by NNPDF2.0 (updates of the CTEQ (CT10 [9]) and MSTW ${ }^{2}$ PDFs will include them). The NNPDF2.0 set does not use a general-mass variable flavor number scheme (the NNPDF2.1 PDF set, which does use a general-mass variable flavor number scheme is currently being finalized ${ }^{3}$ ), but the alternative method which NNPDF use for determining PDF uncertainties provide important independent information.

Other PDF sets, GRJ08, ABKM09 and HERAPDF1.0 are useful for cross checks and specific studies. For example, HERAPDF1.0 allows a study of the theoretical uncertainties related to the charm mass treatment. The $\alpha_{s}$ uncertainties (for the PDFs) can be evaluated by taking a range of \pm 0.0012 for $68 \%$ c.l. (or \pm 0.002 for $90 \%$ c.l.) from the preferred central value for CTEQ and NNPDF. The total PDF $+\alpha_{s}$ uncertainty can then be evaluated by adding the variations in PDFs due to $\alpha_{S}$ uncertainty in quadrature with the fixed $\alpha_{S}$ PDF uncertainty (shown to correctly incorporate correlations in the quadratic error approximation) or, for NNPDF, more efficiently taking a gaussian distribution of PDF replicas corresponding to different values of $\alpha_{s}$. For MSTW the PDF+ $\alpha_{s}$ uncertainties should be evaluated using their prescription which better accounts for correlations between the PDF and $\alpha_{s}$ uncertainties when using the MSTW dynamical tolerance procedure for uncertainties. Adding the $\alpha_{S}$ uncertainty in quadrature for MSTW can be used as a simplification but generally gives slightly smaller uncertainties. So the prescription for NLO is as follows:

- For the calculation of uncertainties at the LHC, use the envelope provided by the central values and PDF $+\alpha_{s}$ errors from the MSTW08, CTEQ6.6 and NNPDF2.0 PDFs, using each group's prescriptions for combining the two types of errors. We propose this definition of an envelope because the deviations between the predictions are as large as their uncertainties. As a central value, use the midpoint of this envelope. We recommend that a $68 \%$ c.l. uncertainty envelope be calculated and the $\alpha_{s}$ variation suggested is consistent with this. Note that the CTEQ6.6 set has uncertainties and $\alpha_{s}$ variations provided only at $90 \%$ c.l. and thus their uncertainties should be reduced by a factor of 1.645 for $68 \%$ c.l.. Within the quadratic approximation, this procedure is completely correct.

\subsection{NNLO prescription}

At NNLO, base predictions on the most widely used NNLO PDF, MSTW2008. There seems to be no reason to believe that the spread in predictions of the global fits, i.e. MSTW, CTEQ and NNPDF, will diminish significantly at NNLO compared to NLO, where this spread was somewhat bigger than the uncertainty from each single group. Hence, at NNLO the uncertainty obtained from

\footnotetext{
${ }^{2}$ The MSTW presentation at the DIS 2010 workshop [10] can be consulted to assess the effects of these data.

${ }^{3}$ The NNPDF presentation at the DIS 2010 workshop [11] can be consulted to assess the effects of these corrections.
} 
MSTW alone should be expanded to some degree. It seems most appropriate to do this by multiplying the MSTW uncertainty at NNLO by the factor obtained by dividing the full uncertainty obtained from the envelope of MSTW, CTEQ and NNPDF results at NLO by the MSTW uncertainty at NLO. In all cases the $\alpha_{s}$ uncertainty should be included. We note that in most cases so far examined for the LHC running at $7 \mathrm{TeV}$ centre of mass energy this factor of the envelope divided by the MSTW uncertainty is quite close to 2, and this factor can be used as a short-hand prescription.

Since there are NNLO PDFs obtained from less global fits by the ABKM, GJR and HERAPDF groups, these can be compared with the above procedure, bearing in mind that there may be kinematic regions where the absence of data, or in the GJR case the theoretical constraint applied to the input, may lead to PDFs and predictions differing significantly from those in a fully global fit. So the prescription at NNLO is:

- As a central value, use the MSTW08 prediction. As an uncertainty, take the same percentage uncertainty on this NNLO prediction as found using the NLO uncertainty prescription given above.

\section{Summary}

The PDF4LHC recommendation summarizes the best of our understanding on PDFs and the associated experimental and theoretical uncertainties. The recommendation is expected to evolve when new experimental sets and new PDF determinations become available.

\section{References}

[1] S. Alekhin, J. Blumlein, S. Klein and S. Moch, Phys. Rev. D 81 (2010) 014032 [arXiv:0908.2766 [hep-ph]].

[2] S. Alekhin, J. Blumlein and S. Moch, arXiv:1007.3657 [hep-ph].

[3] P. M. Nadolsky et al., Phys. Rev. D 78 (2008) 013004 [arXiv:0802.0007 [hep-ph]].

[4] M. Gluck, P. Jimenez-Delgado and E. Reya, Eur. Phys. J. C 53 (2008) 355 [arXiv:0709.0614 [hep-ph]].

[5] F. D. Aaron et al. [H1 Collaboration and ZEUS Collaboration], JHEP 1001 (2010) 109 [arXiv:0911.0884 [hep-ex]].

[6] A. D. Martin, W. J. Stirling, R. S. Thorne and G. Watt, Eur. Phys. J. C 63 (2009) 189 [arXiv:0901.0002 [hep-ph]].

[7] R. D. Ball, L. Del Debbio, S. Forte, A. Guffanti, J. I. Latorre, J. Rojo and M. Ubiali, Nucl. Phys. B 838, 136 (2010) [arXiv:1002.4407 [hep-ph]].

[8] http://mcfm.fnal.gov.

[9] H. L. Lai, M. Guzzi, J. Huston, Z. Li, P. M. Nadolsky, J. Pumplin and C. P. Yuan, arXiv:1007.2241 [hep-ph].

[10] R. S. Thorne, arXiv:1006.5925 [hep-ph].

[11] J. Rojo et al., arXiv:1007.0354 [hep-ph]. 